\title{
Slowly-varying Added Resistance in Irregular Waves in Consideration of Higher Order Components
}

\author{
by $\quad$ Mariko Kuroda ${ }^{*}$, Member Takagi**, Member
}

\begin{abstract}
Summary
So far, added resistance in irregular waves is generally evaluated as a mean value in short term by means of the calculation in frequency-domain. The power spectrum of waves and the frequency response of added resistance in regular waves are used in the calculation, and the added resistance in regular waves is assumed to be proportional to square of wave height based on the 2nd-order wave theory. However, it has been found from tank tests that the added resistance in regular waves is not always proportional to the square of wave height. The major reason is considered that the influence of the hull form above the waterline on the added resistance in waves varies depending on a wave height. Therefore, it is not clear if the 2nd-order theory gives a good estimation for the slowly-varying added resistance in irregular waves.

In this paper, higher order components are considered to estimate the slowly-varying added resistance in irregular waves with the wave height effect. As a result, the slowly-varying added resistance in waves with the consideration of wave height effect is mathematically expressed as the 4th-order component. The estimated results are compared with tank tests, and it has been concluded that the 4th-order component greatly contributed to improvement of the accuracy.
\end{abstract}

\section{Introduction}

The accurate evaluation of ship performance in actual seas is demanded according to the spreading introduction of the onboard measurement system ${ }^{\text {e.g.1), 2) }}$ to actual ships, which enables us to obtain the information for ship performance, engine and encountered weather condition in real time. Added resistance in waves is one of the major components for the evaluation of ship performance in actual seas, so that the accurate calculation of added resistance in waves leads to the precise estimation of ship performance.

Since ocean waves have irregularities with frequencies, wave heights and wave directions, added resistance in irregular waves is generally expressed with the wave spectrum that corresponds to statistical values in short term and the frequency response of added resistance in regular waves. In the calculation, added resistance in regular waves is assumed to be proportional to square of wave height based on the 2nd-order theory. However, it is known from tank tests that it is not always applicable since there are influences of wave height depending on the ship shape above the waterline. Therefore, a direct time-averaged value of added resistance in irregular waves usually does not agree with the mean value of the conventional frequency-domain calculation.

In addition, the variation of added resistance in irregular waves has not been considered since a method for tank tests in irregular waves was not well established to catch the slowly-varying added resistance accurately because of the

\footnotetext{
* National Maritime Research Institute, National Institute of Maritime, Port and Aviation Technology

** The University of Tokyo
}

Received 17/July/2018 appended restoring force which is supposed to affect the slowly-varying added resistance in tank experiment. The appended restoring force is given to a ship model by a torque motor or by a spring to keep ship motion in a certain range when ships do not have their own restoring force. It has been pointed out by Kobayashi ${ }^{3}$ that the appended restoring force have an influence on measured values. Kuroda et al. ${ }^{4)}$ have presented the analysis method for tank tests of added resistance in irregular waves, and showed that the influence of the appended restoring force can be decreased by the correction of the inertia force.

On the other hand, the calculation method for a slowly-varying drifting force in irregular waves has been well established in the offshore engineering for the estimation of mooring forces of an offshore structure. Expanding this method, the calculation method for a slowly-varying added resistance in irregular waves is proposed in this paper. It is shown that the slowly-varying added resistance in irregular waves is expressed as a summation of quadratic frequency response of added resistance in regular waves, and also the effect of wave height is considered as the higher order frequency response. The Newman approximation $^{5}$, which has been used for the calculation of the slowly-varying drifting force of a moored offshore structure, is also employed in the present calculation to simplify the numerical procedure. The adequacy of the method is confirmed through the comparison with experimental results.

\section{Theoretical Background for the Calculation of} Added Resistance in Irregular Waves

Added resistance in irregular waves is conventionally evaluated as a mean value in short term, which is calculated with the wave spectrum and the frequency response of added resistance in regular waves. Eq. (1) is the expression for added resistance in short-crested irregular waves, and Eq. (2) is that for added resistance in long-crested irregular waves. Here, $R_{A W}$ is the 
added resistance in regular waves, $\omega$ is the wave angular frequency, $\alpha$ is the wave direction ( $\alpha=0$ means head waves.), $V$ is the ship speed, $\zeta_{a}$ is the wave amplitude, $E(\omega, \alpha)$ is the directional wave spectrum, and $S(\omega)$ is the wave power spectrum.

$$
\begin{gathered}
R_{\text {wave }}=2 \int_{0}^{2 \pi} \int_{0}^{\infty} \frac{R_{A W}(\omega, \alpha ; V)}{\zeta_{a}{ }^{2}} E(\omega, \alpha) d \omega d \alpha \\
R_{A W L}=2 \int_{0}^{\infty} \frac{R_{A W}(\omega)}{\zeta_{a}{ }^{2}} S(\omega) d \omega
\end{gathered}
$$

For the calculation of $R_{A W}$, a practical method ${ }^{6)}$ in which Maruo's theory ${ }^{7)}$ combined with a correction for diffraction term using the experimental parameter is employed. Here, $R_{A W}$ is composed of the added resistance due to primary induced by ship motion $R_{A W M}$ and the added resistance for correction of diffraction component $R_{A W R}$.

$$
R_{A W}=R_{A W M}+R_{A W R}
$$

$R_{A W M}$ is calculated by Maruo's theory with the ship motion. $R_{A W R}$ is calculated by the equation that comprises the bluntness coefficient $B_{f}$, the coefficient of draft and frequency $\alpha_{d}$ and the effect of advance speed $\left(1+C_{U} F_{n}\right)$, where $C_{U}$ is obtained by tank tests in waves or by an empirical formula.

On the other hand, since the irregular waves are expressed as the superposition of regular waves, the slow variation, which corresponds to the difference frequency of component regular waves, is occurred.

A slowly-varying added resistance in irregular waves is generally represented by the 2 nd-order response of component waves as shown in Eq. (4), where the irregular waves $\zeta$ are expressed as Eq. (5), $t$ is the time, $A_{i}$ is the complex amplitude including random phase, $\omega_{e i}$ is the angular frequency of encountered waves, $A_{i}$ and $\omega_{e i}$ correspond to the $i$-th component waves, $M_{i,-j}\left(\omega_{e i}, \omega_{e j}\right)$ is the quadratic transfer function of difference frequencies, and $*$ denotes the complex conjugate.

$$
\begin{gathered}
\Delta R(t)=\operatorname{Re} \sum_{i} \sum_{j} A_{i} A_{j}^{*} M_{i,-j}\left(\omega_{e i}, \omega_{e j}\right) e^{i\left(\omega_{e i}-\omega_{e j}\right) t} \\
\zeta(t)=\operatorname{Re} \sum_{i} A_{i} e^{i \omega_{e i} t}
\end{gathered}
$$

The Newman approximation is presented as Eq. (6), where it is assumed that the contribution of diagonal term is dominant if the wave spectrum is narrow band, and thus the component of quadratic transfer functions is approximated by the diagonal term.

$$
M_{i,-j}\left(\omega_{e i}, \omega_{e j}\right) \cong M_{i,-j}\left(\omega_{e i}, \omega_{e i}\right)
$$

If the Newman approximation is employed, Eq. (4) is approximated as

$$
\Delta R(t) \cong \operatorname{Re} \sum_{i} \sum_{j} A_{i} A_{j}^{*} M_{i,-i}\left(\omega_{e i}, \omega_{e i}\right) e^{i\left(\omega_{e i}-\omega_{e j}\right) t}
$$

The quadratic transfer functions for the difference frequency $M_{i,-i}\left(\omega_{e i}, \omega_{e i}\right)$ equals to the frequency response of added resistance in regular waves.

$$
M_{i,-i}\left(\omega_{e i}, \omega_{e i}\right)=\frac{R_{A W}\left(\omega_{e i}\right)}{\zeta_{a}^{2}}
$$

Thus, the slowly-varying added resistance in irregular waves is evaluated from the added resistance in regular waves obtained by the conventional calculation method. However, the wave height effect should be considered as mentioned previously. This effect is discussed in the next section.

\section{Added Resistance in Waves with Higher Order Components}

The wave height effect on the added resistance in regular waves has been known empirically ${ }^{\text {e.g. } 8), 9)}$, and that on the added resistance in irregular waves has also been pointed out ${ }^{\text {e.g. } 2), 9)}$. The incorporation of the wave height effect as higher-order components is discussed here.

\section{1 Regular Waves}

It is well-known that the first-order regular waves are expressed as Eq. (9). Here, $A_{i}^{r}$ is the wave amplitude, $\omega_{e i}$ is the encountered wave angular frequency, $\delta_{i}$ is the phase difference, and the subscript $i$ means $i$-th frequency, though regular waves have only one frequency component. It is also noted that $\delta_{i}$ is not necessary to be considered for regular waves.

$$
\begin{aligned}
& \zeta_{i}=A_{i}^{r} \cos \alpha_{i} \\
& \alpha_{i}=\omega_{e i} t+\delta_{i} \\
& \alpha_{i}=\omega_{e i} t \text { for regular waves }
\end{aligned}
$$

Components up to 4th-order hydrodynamic forces in waves are expressed with the transfer functions $M$.

$$
\begin{aligned}
\Delta R_{i}(t) & =M_{i} A_{i}^{r} \cos \left(\alpha_{i}\right) \\
& +M_{i, \pm i} A_{i}^{r 2} \cos \left(\alpha_{i} \pm \alpha_{i}\right) \\
& +M_{i, \pm i, \pm i} A_{i}^{r 3} \cos \left(\alpha_{i} \pm \alpha_{i} \pm \alpha_{i}\right) \\
& +M_{i, \pm i, \pm i, \pm i} A_{i}^{r 4} \cos \left(\alpha_{i} \pm \alpha_{i} \pm \alpha_{i} \pm \alpha_{i}\right)
\end{aligned}
$$

where, $M_{i, \pm i}=M_{i, \pm i}\left(\omega_{e i}, \omega_{e i}\right), M_{i, \pm i, \pm i}=M_{i, \pm i, \pm i}\left(\omega_{e i}, \omega_{e i}, \omega_{e i}\right)$ and $M_{i, \pm i, \pm i, \pm i}=M_{i, \pm i, \pm i, \pm}\left(\omega_{e i}, \omega_{e i}, \omega_{e i}, \omega_{e i}\right)$, and \pm denotes summation of all possible combinations.

However, the added resistance in regular waves usually means the time-average for the encountered wave period. Considering the time-average of Eq. (12), only the constant terms 
remain in the 2nd-order and 4th-order components and the 1st-order and 3rd-order components are vanished as Eq. (13), where Eq. (14) is satisfied.

$$
\begin{aligned}
& \overline{\Delta R_{i}}=M_{i,-i} A_{i}^{r 2}+3 M_{i, i,-i,-i} A_{i}^{r 4} \\
& M_{i, i,-i,-i}=M_{i,-i, i,-i}=M_{i,-i, i-i}
\end{aligned}
$$

In the section 2, the transfer function $M_{i,-i}$ is expressed as the frequency response of added resistance in regular waves, which is obtained by the conventional calculation method. Similarly, the transfer function $M_{i, i,-i,-i}$ is expressed as the 4 th-order component of added resistance in regular waves $R_{A W}^{(4)}$.

$$
3 M_{i, i,-i,-i}=\frac{R_{A W}^{(4)}}{\zeta_{a}^{4}}
$$

where, $R_{A W}^{(4)}$ is obtained from the measurement of added resistance in regular waves as a component which is proportional to the 4th-power of wave height.

\section{2 Irregular Waves}

Similarly to regular waves, higher order components of hydrodynamic forces in irregular waves are considered. The first order components of irregular waves are expressed by Eq. (16), which corresponds to Eq. (5), though $A_{i}^{r}$ is a real value and $\alpha_{i}$ includes the random phase $\delta_{i}$.

$$
\zeta(t)=\sum_{i} A_{i}^{r} \cos \alpha_{i}
$$

Higher order components up to 4th-order hydrodynamic forces in irregular waves are expressed as

$$
\begin{aligned}
& \Delta R(t) \\
& =\sum_{i} A_{i}^{r} M_{i} \cos \alpha_{i} \\
& +\sum_{i} \sum_{j} A_{i}^{r} A_{j}^{r} M_{i, \pm j} \cos \left(\alpha_{i} \pm \alpha_{j}\right) \\
& +\sum_{i} \sum_{j} \sum_{k} A_{i}^{r} A_{j}^{r} A_{k}^{r} M_{i, \pm j, \pm k} \cos \left(\alpha_{i} \pm \alpha_{j} \pm \alpha_{k}\right) \\
& +\sum_{i} \sum_{j} \sum_{k} \sum_{l} A_{i}^{r} A_{j}^{r} A_{k}^{r} A_{l}^{r} M_{i, \pm j, \pm k, \pm l} \cos \left(\alpha_{i} \pm \alpha_{j} \pm \alpha_{k} \pm \alpha_{l}\right)
\end{aligned}
$$

Conventional added resistance in waves is the 2nd-order value, and the slowly-varying component of the 2 nd-order value is the frequency-difference component of the second term in Eq. (17). In the sub-section 2.2, the Newman approximation is applied to the 2nd-order component, and the slowly-varying added resistance in irregular waves is presented by the frequency response of added resistance in regular waves as Eq. (7).

Here, the concept of the Newman approximation is expanded to the higher order component. In addition, since the spectrum band of waves is narrow, the 3rd-order slowly-varying component is very small compared to the 2 nd-order or 4 th-order components. Therefore, the 2nd-order term and the 4th-order term remain as slowly-varying components similarly to the case of the added resistance in regular waves. After applying the concept of Newman approximation, the slowly-varying added resistance in irregular waves $\Delta \widetilde{R}(t)$ is represented by Eq. (18).

$$
\begin{aligned}
& \Delta \widetilde{R}(t) \\
\cong & \sum_{i} \sum_{j} A_{i}^{r} A_{j}^{r} M_{i,-i} \cos \left(\alpha_{i}-\alpha_{j}\right) \\
& +\sum_{i} \sum_{j} \sum_{k} \sum_{l} A_{i}^{r} A_{j}^{r} A_{k}^{r} A_{l}^{r} 3 M_{i, i,-i,-i} \cos \left(\alpha_{i}+\alpha_{j}-\alpha_{k}-\alpha_{l}\right)
\end{aligned}
$$

The 2nd-order transfer function $M_{i,-i}$ corresponds to the first component of Eq. (13), and the 4th-order transfer function $M_{i, i,-i,-i}$ corresponds to the second component of Eq. (13). Thus, the slowly-varying added resistance in irregular waves is expressed with the 2nd-order and the 4th-order frequency responses of added resistance in regular waves.

\section{Incorporation of the Wave Height Effect as the 4th-order Component}

In this section, a slowly-varying added resistance in irregular waves is estimated by the method described in the section 3 . At first, how to incorporate the effect of wave height into the mathematical expression as the 4th-order component is presented. Then, the effect of wave height is obtained by using tank test results.

Here, test results by Kuroda et al. ${ }^{4)}$ for VLCC model (length between perpendiculars $L_{p p}: 4.16 \mathrm{~m}$, ship breadth $B: 0.77 \mathrm{~m}$, draft $d: 0.26 \mathrm{~m}$, longitudinal radius of gyration $k_{y y} / L_{p p}: 0.25$ ) are used.

\section{1 Expression of the 4th-order Component}

The effect of wave height on added resistance in regular waves is shown in Fig. 2 for the VLCC model at $F_{n}=0.141$ in head waves. Here, $K_{A W}$ is the coefficient of added resistance in waves calculated by Eq. (19), $F_{n}$ is the Froude number obtained by Eq. (20), $\rho$ is the fluid density, $g$ is the gravitational acceleration, $\zeta_{a}$ is the wave amplitude, $V$ is the speed, $\lambda$ is the wave length, and the dotted line and the solid line in Fig. 2 express the calculated results from Eq. (3) shown in the section 2.

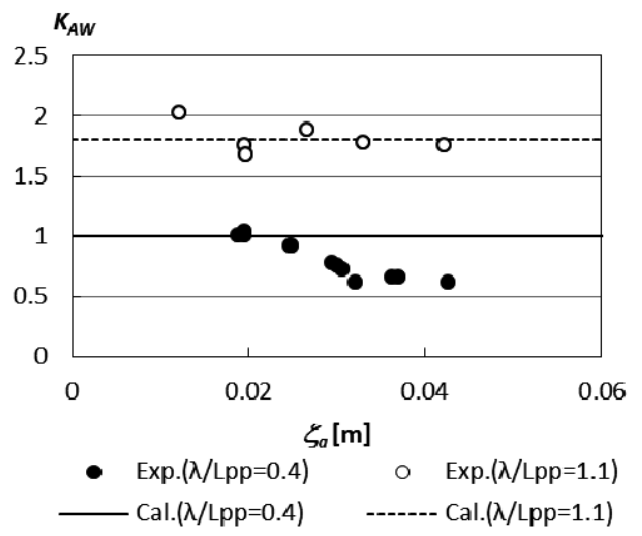

Fig. 2 Effect of wave height on added resistance in regular waves

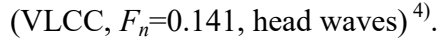




$$
\begin{gathered}
K_{A W}=\frac{R_{A W}}{4 \rho g \zeta_{a}^{2}\left(B^{2} / L_{p p}\right)} \\
F_{n}=\frac{V}{\sqrt{g L_{p p}}}
\end{gathered}
$$

Fig. 2 indicates the presence of the wave height effect especially in short wave length $\left(\lambda L_{p p}=0.4\right)$. Here, the relation between the added resistance in regular waves and the wave amplitude $\zeta_{a}$ is illustrated in Fig. 3. The fitted curves with the quadratic expression of $\zeta_{a}$ are shown by dashed lines, and the fitted curves with the 2nd and 4th-order components of $\zeta_{a}$ are illustrated by solid lines, which correspond to the expression by Eq. (13). Fig. 3(a) indicates that the introduction of the 4th-order component makes the agreement with the experimental data better. Similarly, Fig. 3(b) indicates the relation for $\lambda / L_{p p}=1.1$. Since the added resistance in regular waves in the case of $\lambda / L_{p p}=1.1$ shows much smaller the wave height effect compared to the case of $\lambda L_{p p}=0.4$ as shown in Fig. 2 , the agreement of the fitted curves is little different. The estimation accuracy of added resistance in regular waves is expected to be improved by introducing the 4 th-order components.

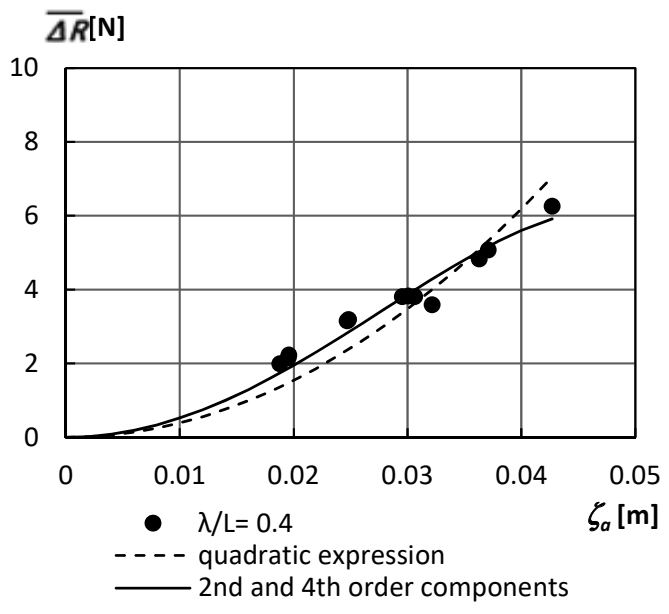

(a) $\lambda / L_{p p}=0.4$

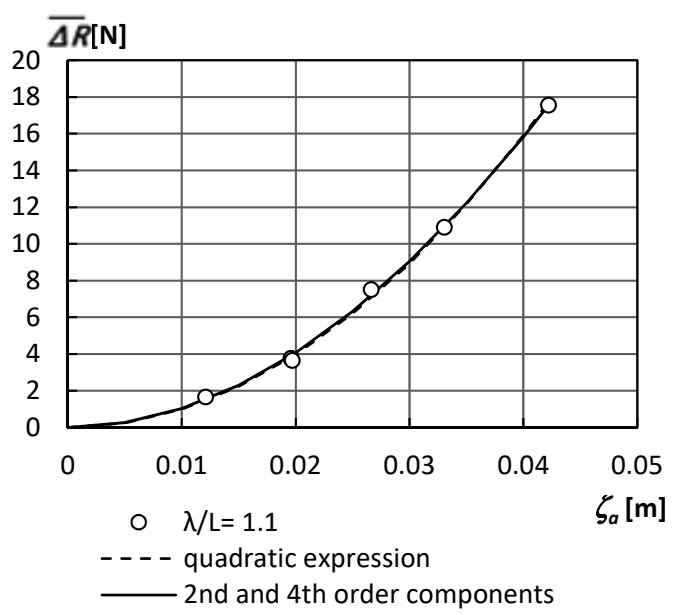

(b) $\lambda / L_{p p}=1.1$

Fig. 3 The expression of added resistance in regular waves (VLCC, $F_{n}=0.141$, head waves).
Fig. 4 indicates the calculated results for the 2nd-order frequency response of added resistance in waves by the method described in Section 2. The parameter $C_{U}$ for the calculation of the 2nd-order frequency response was obtained from a tank test in short regular waves of wave height $3 \mathrm{~m}$ in full scale $(0.0385 \mathrm{~m}$ in model scale). Therefore, the agreement between calculated results and experimental results is good around $\zeta_{\text {am }} \cong 0.02 \mathrm{~m}$ in Fig . 2 .

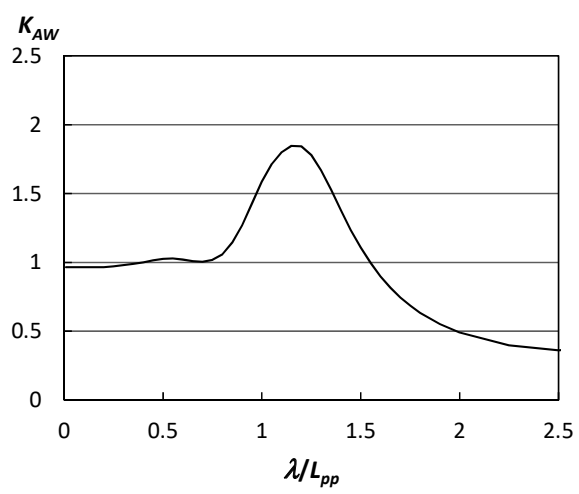

Fig. 4 2nd-order frequency response of added resistance in waves (VLCC, $F_{n}=0.141$, head waves).

The difference between the added resistance in regular waves obtained by the experiment in Fig. 3 and the calculated added resistance in regular waves $R_{A W}^{c a l}$ by the conventional method shown in Fig. 4 is shown in Fig. 5 as $\delta \Delta R$. Although $\delta \Delta R$, which includes error in the calculation by the method described in Section 2.1, should be divided into the error in the 2nd-order component and the 4th-order component, $\delta \Delta R$ is expressed as the 4th-order component of $\zeta_{a}$ since the error of the conventional method at $\lambda / L_{p p}=0.4$ and 1.1 is almost zero at $\zeta_{a m} \cong 0.02$. From Eq. (13), the difference $\delta \Delta R$ is expressed by the quartic formula as shown in Eq. (21), where $A_{i}^{r}$ is equal to $\zeta_{\mathrm{a}}$ in regular waves. The fitted curves by the quartic formula are shown in Fig. 5.

$$
\delta \Delta R=\overline{\Delta R}-R_{A W}^{c a l} \cong \overline{\Delta R}-M_{i,-i} A_{i}^{r 2}=3 M_{i, i,-i,-i} A_{i}^{r 4}=R_{A W}^{(4)}
$$

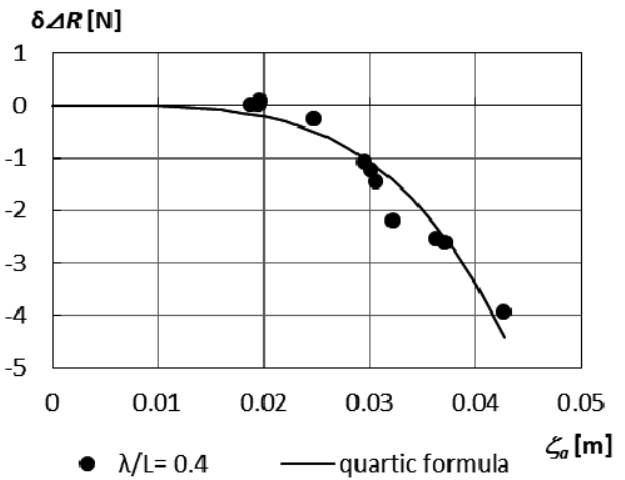

(a) $\lambda / L_{p p}=0.4$ 


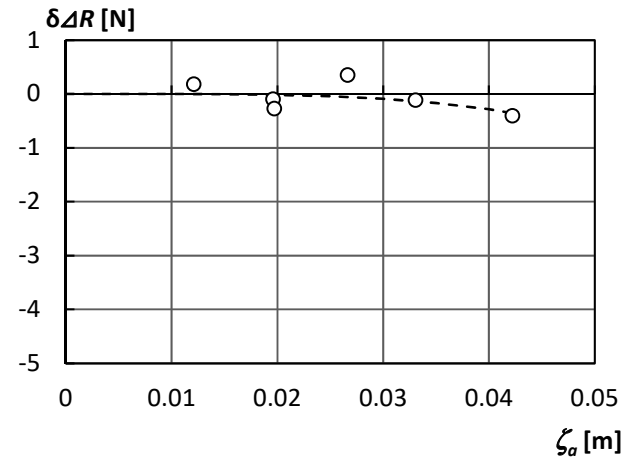

○ $\lambda / L=1.1 \quad-\cdots$ quartic formula

(b) $\lambda / L_{p p}=1.1$

Fig. 5 Expression of the 4th-order component (VLCC, $F_{n}=0.141$, head waves).

With the obtained transfer functions $3 M_{i, i, i, i, i}$ and the calculated 2nd-order components of added resistance shown in Fig.4, the relation between $\overline{\Delta R}$ and $\zeta_{\mathrm{a}}$ is calculated as shown in Fig. 6. It is found that the estimated line with the 4th-order components based on the conventional method is close to the fitted curve of experimental results and represents the good agreement with experimental data. In Fig. 6, the estimated curves by the calculation method described in Section 2.1 are shown by dashed lines as reference.

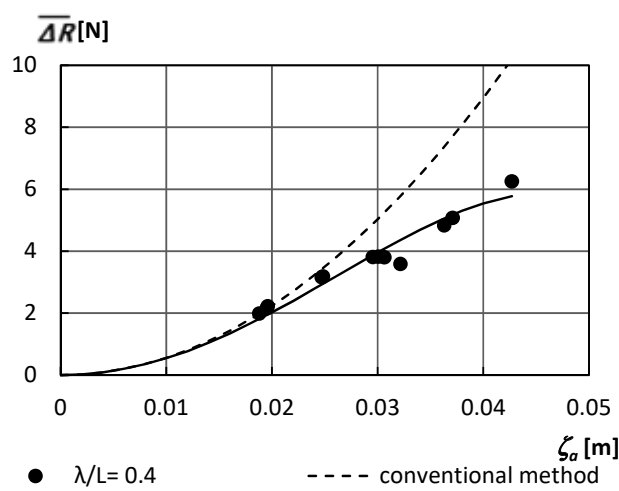

with 4 th order components based on the conventional method

(a) $\lambda / L_{p p}=0.4$

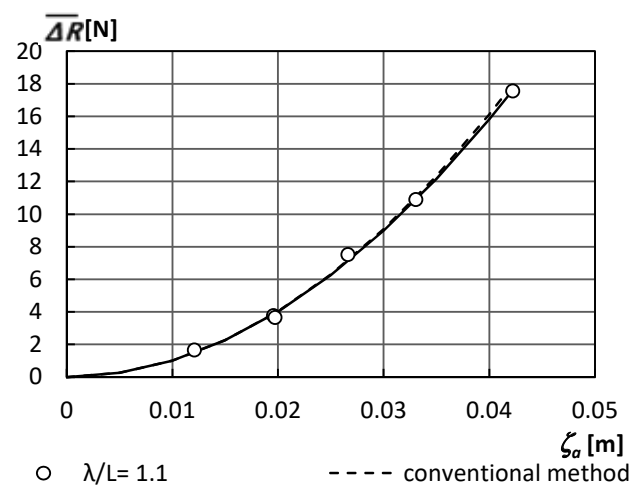

with 4th order components based on the conventional method

(b) $\lambda / L_{p p}=1.1$

Fig. 6 Relation between added resistance in waves and wave amplitude.(VLCC, $F_{n}=0.141$, head waves).
For the estimation of the slowly-varying added resistance in irregular waves, a frequency response for the 4th-order components is required as well as that for 2 nd-order components. However, we have 4th-order components only for $\lambda L_{p p}=0.4$ and 1.1 , thus for other wave frequency, the liner interpolation and the allongement of end-point values are applied in consideration for the frequency spectrum of irregular waves. For short wave length, the allongement of an end-point value is applied analogically with the frequency response of 2 nd-order components. For long wave length, the allongement of an end-point value is also applied since the frequency spectrum of irregular waves for the calculation is not significant for long wave length $\left(\lambda / L_{p p} \geq 1.1\right)$ as shown in Fig. 8 .

From the above, the frequency response of 4th-order components of added resistance in regular waves is assumed as shown in Fig. 7. Here, the 4th-order added resistance in regular waves $K_{A W}^{(4)}$ is normalized by Eq. (22).

$$
K_{A W}^{(4)}=\frac{R_{A W}^{(4)}}{16 \rho g \zeta_{a}^{4}\left(B / L_{p p}^{2}\right)}
$$

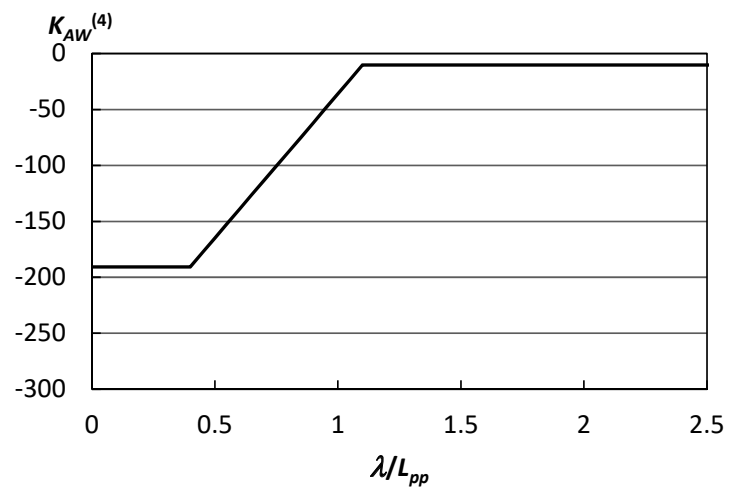

Fig. 74 th-order frequency response of added resistance in waves (VLCC, $F_{n}=0.141$, head waves).

Now, the transfer functions $M_{i,-i}$ and $M_{i, i,-i,-i}$ are obtained from the frequency response shown in Fig. 4 and Fig. 7, thus the slowly-varying added resistance in irregular waves can be calculated by Eq. (18).

\section{2 Calculation of the Slowly-varying Added Resistance in Irregular Waves}

The slowly-varying added resistance in irregular waves is estimated by using the method presented in previous sections, and the adequacy of the estimation method is confirmed through the comparison with test results by Kuroda et al. ${ }^{4)}$ in the condition of the maximum appended restoring force for a longitudinal motion. Measured irregular waves are long-crested head irregular waves, and the power spectrum is shown in Fig. 8. The significant wave height $H_{1 / 3}$ and the mean wave period $T_{01}$ calculated from the measured power spectrum are $H_{1 / 3}=0.0677 \mathrm{~m}, T_{01}=1.09 \mathrm{~s}$, which are close to Beaufort scale 8 in full scale. 


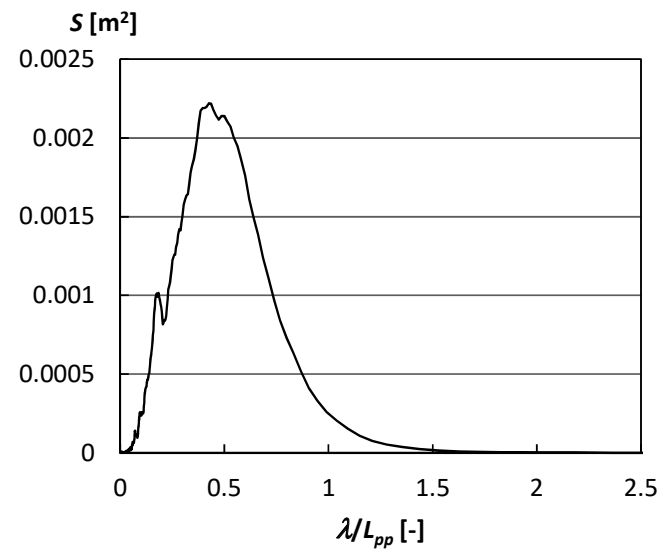

Fig. 8 Power spectrum.

Since the time history of measured waves contains high and low frequency variations which can be neglected for the estimation of the slowly-varying added resistance, component waves ranging between $\lambda L_{p p}=0.10 \sim 1.17$ are used for the calculation. Time history of measured waves is shown in Fig. 9.

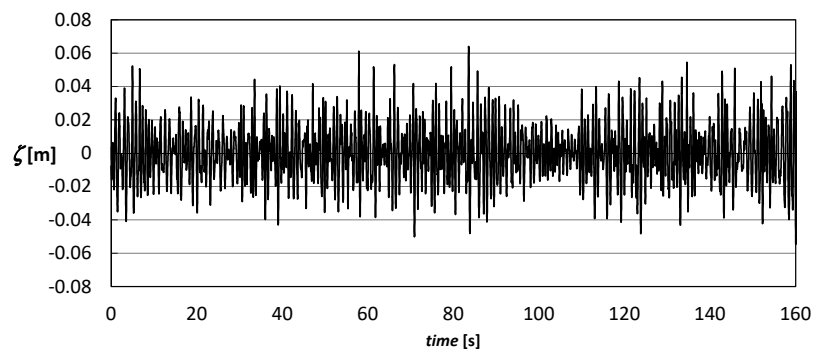

Fig. 9 Time history of measured long-crested irregular waves

$$
\left(\mathrm{VLCC}, F_{n}=0.141\right)^{4)} \text {. }
$$

The calculated results of the 2nd-order slowly-varying added resistance in long-crested irregular waves $\Delta \widetilde{R}$ are shown in Fig. 10 (a). It is apparent that the calculated result over-estimates the experimental result especially when the wave height is locally high. The reason is considered to be the effect of wave height. The calculated result including the 4th-order component is shown in Fig. 10(b), which shows the good agreement with the experimental result especially at locally high waves for instance time $=80 \mathrm{~s}$ and time $=120 \mathrm{~s}$.

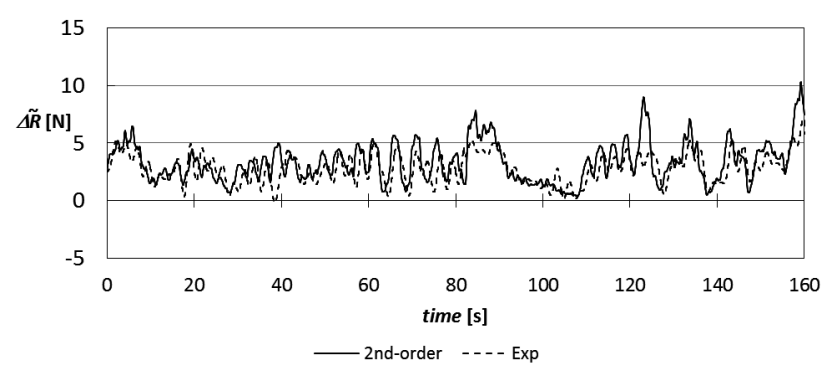

(a) Calculation with the 2 nd-order frequency response.

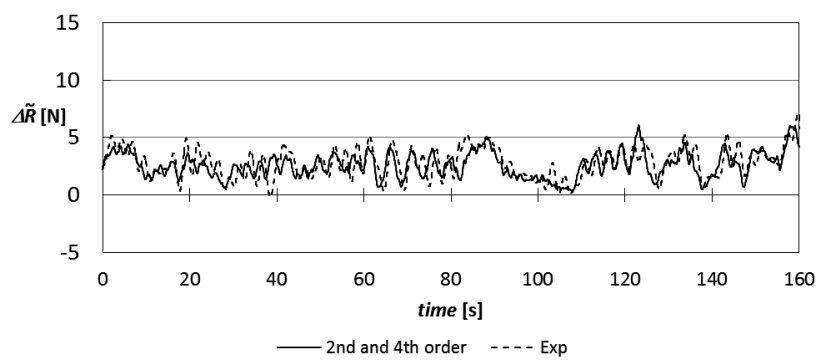

(b) Calculation with the 2nd and 4th-order frequency response.

Fig. 10 A slowly-varying added resistance in long-crested irregular waves (VLCC, $F_{n}=0.141$ ).

In order to evaluated the result quantitatively, the root mean square error $(\overline{R M S E})$ is calculated as shown in Table 1. Here, $\overline{R M S E}$ is normalized by time-average of experimental time history. From Fig. 8 and the comparison of $\overline{R M S E}$, it is found that the agreement with the experimental value is improved by incorporating the effect of wave height as the 4th-order component into the calculation of slowly-varying in added resistance in irregular waves.

Table 1 Comparison of the root mean square error.

\begin{tabular}{|c|c|c|}
\hline & Cal. with 2nd component & $\begin{array}{c}\text { Cal. with 2nd and 4th } \\
\text { components }\end{array}$ \\
\hline$\overline{R M S E}$ & 0.68 & 0.36 \\
\hline
\end{tabular}

The time-average of added resistance in irregular waves is considered. Since the average of the 2nd-order component, which corresponds to the first term in Eq. (18), is obtained by summing up the component of $\alpha_{i}=\alpha_{j}$, the averaged value is expressed by Eq. (23). Here, the first term in Eq. (18) is represented by $\Delta \widetilde{R}^{(2)}(t)$. The relation between the wave spectrum and the wave amplitude is expressed as Eq. (24). Eq. (23) equals to the conventional expression of added resistance in long-crested irregular waves shown by Eq. (2).

$$
\begin{aligned}
\overline{\Delta \widetilde{R}^{(2)}(t)} & =\sum_{i} A_{i}^{r 2} M_{i,-i}\left(\omega_{e i}, \omega_{e i}\right) \\
& =2 \sum_{i} \frac{R_{A W}\left(\omega_{e i}\right)}{\zeta_{a}^{2}} S\left(\omega_{e i}\right) \Delta \omega_{e i} \\
\frac{1}{2} A_{i}^{r 2} & =S\left(\omega_{e i}\right) \Delta \omega_{e i}
\end{aligned}
$$

On the contrary, the time-average of the 4th-order component which corresponds to the 2nd term in Eq. (18) is not easy to obtain because we need to consider all cases for $\left(\alpha_{i}+\alpha_{j}\right)=\left(\alpha_{k}\right.$ $\left.+\alpha_{l}\right)$.

The comparison of mean values is shown in Fig. 11 and in Table 2. Here, component waves in the range of $\lambda L_{p p}=0.10 \sim$ 1.17 are used as with the calculation shown in Fig. 10. In Fig. 11, "Exp" is the time-average of experimental time history shown in Fig. 10, "time history $2 \& 4$ " is the time-average of time history calculated with 2nd-order and 4th-order components shown in Fig. 
10 (b), "time history 2" is the time-average of time history calculated only with the 2nd-order component shown in Fig. 10 (a), "spectrum 2" is the calculated value by Eq. (23).

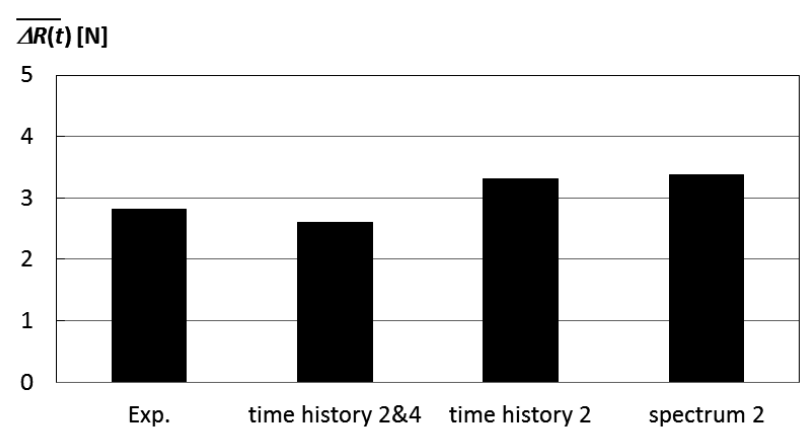

Fig. 11 Mean value of added resistance in long-crested irregular waves (VLCC, $F_{n}=0.141$ ).

Table 2 Comparison of the root mean square error.

\begin{tabular}{|c|c|c|c|c|}
\hline & Exp. & $\begin{array}{c}\text { time history } \\
2 \& 4\end{array}$ & time history & spectrum 2 \\
\hline$\overline{\Delta R}$ & 2.82 & 2.60 & 3.31 & 3.37 \\
\hline$\overline{\Delta R} / \overline{\Delta R_{\text {Exp. }}[\%]}$ & - & -8.0 & 17.4 & 19.4 \\
\hline
\end{tabular}

As mentioned above, "time history 2" and "spectrum 2" consider only the 2nd-order component, thus these values are larger than "Exp", since the effect of wave height is not considered. On the contrary, "time history $2 \& 4$ " considers the 4th-order component as well as the 2nd-order component, therefore it can reflect the wave height effect, and thus it is in good agreement with "Exp", where the difference between calculation "time history 2\&4" and experiment "Exp" is improved in around $10 \%$ from that with "time history 2 "/ "spectrum 2".

\section{Conclusions}

The expression of added resistance in waves in consideration with the 4th-order component to represent the wave height effect and to improve the calculation of time history has been shown. A methodology to use the experimental result of wave height effect on added resistance in regular waves is presented to determine the 4th-order component, and the slowly-varying added resistance in long-crested irregular waves has been estimated. Through the comparison with experimental results for VLCC model, it is concluded that the agreement with experimental results is greatly improved with the consideration of the 4th-order components Since the consideration in this paper is aimed only to the VLCC model in long- crested irregular head waves at a certain speed, it is necessary to confirm the applicability of the method for other kinds of ships, in various wave direction, and at various speeds.

\section{References}

1) H. Ando: Performance Monitoring for Energy Efficient Fleet Operation (in Japanese), Journal of the Society of Instrument and Control Engineers, vol.50-6, pp. 398-404, 2011.

2) H. Yoshida, H. Orihara, K. Yamasaki: Voyage Support System "Sea-Navi ${ }^{\circledR}$ " (in Japanese), JFE Technical Report, vol.32, pp. 87-90, 2013.

3) M. Kobayashi: Consideration on the Added Resistance and Speed Drop of a Ship with Forward Speed (in Japanese), Conference proceedings of the Japan Society of Naval Architects and Ocean Engineers, vol. 4, pp. 251-254, 2007.

4) M. Kuroda, K. Takagi, M. Tsujimoto and J. Fujisawa: Measurement of Added Resistance in Irregular Waves and Estimation of the Long-period Components (in Japanese), Journal of the Japan Society of Naval Architects and Ocean Engineers, Vol. 24, pp. 183-190, 2016.

5) J.N. Newman: Second-order, Slowly-varying Forces on Vessel in Irregular Waves, Proceedings of the Symposium on the Dynamics of Marine Vehicle and Structured in Waves, pp.182-186, 1974.

6) M. Tsujimoto, K. Shibata, M. Kuroda and K. Takagi: A Practical Correction Method for Added Resistance in Waves, Journal of the Japan Society of Naval Architects and Ocean Engineers, Vol. 8, pp. 177-184, 2008.

7) H. Maruo: Resistance in Waves, Research on Seakeeping Qualities of Ships in Japan, The Society of Naval Architects of Japan, vol.8, pp. 67-102, 1963.

8) S. Nakamura, R. Hosoda and S. Naito: Propulsive Performance of a Container Ship in Waves (3rd Report) (in Japanese), Journal of the Kansai Society of Naval Architects, Japan, Vol. 158, pp. 37-46, 1975.

9) H. Yasukawa, A. Matsumoto and S. Ikezoe: Wave Height Effect on Added Resistance in Full Hull Ships in Waves (in Japanese), Journal of the Japan Society of Naval Architects and Ocean Engineers, Vol. 23, pp. 45-54, 2016. 